\title{
Some Fourth Degree Integration Formulas for Simplexes
}

\author{
By A. H. Stroud
}

Abstract. A fourth degree integration formula is given for the $n$-dimensional simplex for $n=3,4,5,6,7,8,10,11,12$. The formula contains $\left(n^{2}+3 n+4\right) / 2$ points.

1. Introduction. We consider integration formulas

$$
\int \cdots \int_{T_{n}}^{\ldots} f\left(x_{1}, \ldots, x_{n}\right) d x_{1} \cdots d x_{n} \simeq \sum_{k=1}^{N} A_{k} f\left(x_{k 1}, \ldots, x_{k n}\right) \text {. }
$$

Here $T_{n}$ is the $n$-simplex whose $n+1$ vertices are

$$
\begin{gathered}
(0,0,0, \ldots, 0,0), \\
(1,0,0, \ldots, 0,0), \\
(0,1,0, \ldots, 0,0), \\
\ldots \\
(0,0,0, \ldots, 0,1) .
\end{gathered}
$$

A formula (1) is said to have degree $d$ if it is exact for all polynomials of degree $\leqslant d$ and if there is at least one polynomial of degree $d+1$ for which it is not exact.

A number of formulas (1) are given by Stroud [4, pp. 306-315]. Other than product formulas there is only one formula of degree four known for all $n \geqslant 3$. This formula (denoted in [4] as $\left.T_{n}: 4-1\right)$ contains $(n+4) ! /(4 ! n !)$ points; it was first given by Lauffer [2]. A fourth degree formula for $T_{2}$ is known with six points [3].

The purpose of this note is to give a fourth degree formula (1) for $n=3,4,5,6$, $7,8,10,11,12$ which contains $N=(n+1)(n+2) / 2+1$ points. For an arbitrary $n$-dimensional region $R_{n}$ it is known that a fourth degree formula must contain at least $(n+1)(n+2) / 2$ points (see [4, p. 118]). For $T_{n}, n \geqslant 3$, we believe that no fourth degree formula exists with $(n+1)(n+2) / 2$ points; if this is true, the formulas given below contain the minimal number of points for fourth degree.

2. Construction of the Formulas. The formulas to be described are invariant under all affine transformations of $T_{n}$ onto itself. We use the notation of [4, p. 307] to describe the points in these formulas. The notation

$$
\left(u_{1}, u_{2}, \ldots, u_{n} ; u_{n+1}\right)
$$

denotes the set of points consisting of the point $\left(u_{1}, u_{2}, \ldots, u_{n}\right)$ and all points which this point maps into under the set of all $(n+1)$ ! affine transformations of $T_{n}$ onto itself. (It will always be true that $u_{1}+u_{2}+\cdots+u_{n+1}=1$.) The coefficients $A_{k}$ in formula (1) which correspond to the points in a set (2) will all have the same value. We seek formulas with points and coefficients as follows:

Received June 12, 1975.

AMS (MOS) subject classifications (1970). Primary 65D30; Secondary 41 A55.

Key words and phrases. Numerical integration, integration formulas, simplexes. 


$$
\begin{array}{ccc}
\text { set of points } & \text { coeff. } & \text { points in set } \\
(r, r, \ldots, r, r ; r) & A & 1 \\
(s, s, \ldots, s, s ; t) & B & n+1 \\
(u, u, \ldots, u, v ; v) & C & n(n+1) / 2
\end{array}
$$

Here $r=1 /(n+1)$ and the point (3) is the centroid of $T_{n}$. Unless $s=t=r$ or $u=v$ $=r$ the union of the sets (3), (4), (5) contains $\left(n^{2}+3 n+4\right) / 2$ distinct points. There are five free parameters $t, v, A, B, C$ to be determined.

THEOREM 1. If formula (1) consists of the points and coefficients (3), (4), (5), and if this formula is exact for the monomials

$$
1, x_{1}^{2}, x_{1}^{3}, x_{1}^{4}, x_{1}^{2} x_{2}^{2}
$$

then the formula is exact for all polynomials of degree $\leqslant 4$.

Proof. The proof is an application of Theorem 3.14-1 of [4, p. 115]. The fact that the formula is exact for all polynomials of degree $\leqslant 3$ is the same as the proof of Theorem 3.14-2 of $[4$, p. 116]. To complete the proof we must only yet show that the formula is exact for $x_{1}^{3} x_{2}, x_{1}^{2} x_{2} x_{3}$ and $x_{1} x_{2} x_{3} x_{4}$. Since the formula is known to be exact for $x_{1}^{3}$ and $x_{1}^{4}$, Theorem 3.14-1 shows it is exact for $x_{1}^{3} x_{2}$. Since it is exact for $x_{1}^{2} x_{2}, x_{1}^{3} x_{2}$ and $x_{1}^{2} x_{2}^{2}$, Theorem 3.14-1 shows it is exact for $x_{1}^{2} x_{2} x_{3}$. Since it is exact for $x_{1} x_{2} x_{3}, x_{1}^{2} x_{2} x_{3}, x_{1} x_{2}^{2} x_{3}$ and $x_{1} x_{2} x_{3}^{2}$, Theorem 3.14-1 shows it is exact for $x_{1} x_{2} x_{3} x_{4}$. This completes the proof.

The equations which must be satisfied if the points and coefficients (3), (4), (5) are to be a formula which is exact for the monomials (6) are the following:

$$
A+(n+1) B+n(n+1) C / 2=I[1]
$$

$$
\begin{aligned}
& A r^{2}+B\left[n s^{2}+t^{2}\right]+n C\left[\frac{(n-1)}{2} u^{2}+v^{2}\right]=I\left[x_{1}^{2}\right], \\
& A r^{3}+B\left[n s^{3}+t^{3}\right]+n C\left[\frac{(n-1)}{2} u^{3}+v^{3}\right]=I\left[x_{1}^{3}\right], \\
& A r^{4}+B\left[n s^{4}+t^{4}\right]+n C\left[\frac{(n-1)}{2} u^{4}+v^{4}\right]=I\left[x_{1}^{4}\right]
\end{aligned}
$$

$A r^{4}+B\left[(n-1) s^{4}+2 s^{2} t^{2}\right]$

$$
\begin{aligned}
& +C\left[\frac{(n-1)(n-2)}{2} u^{4}+2(n-1) u^{2} v^{2}+v^{4}\right]=I\left[x_{1}^{2} x_{2}^{2}\right] \text {, } \\
& I\left[x_{1}^{\alpha} x_{2}^{\beta}\right] \equiv \int \underset{T_{n}}{\int} \int_{1}^{\alpha} x_{1}^{\alpha} d x_{1} \cdots d x_{n} .
\end{aligned}
$$




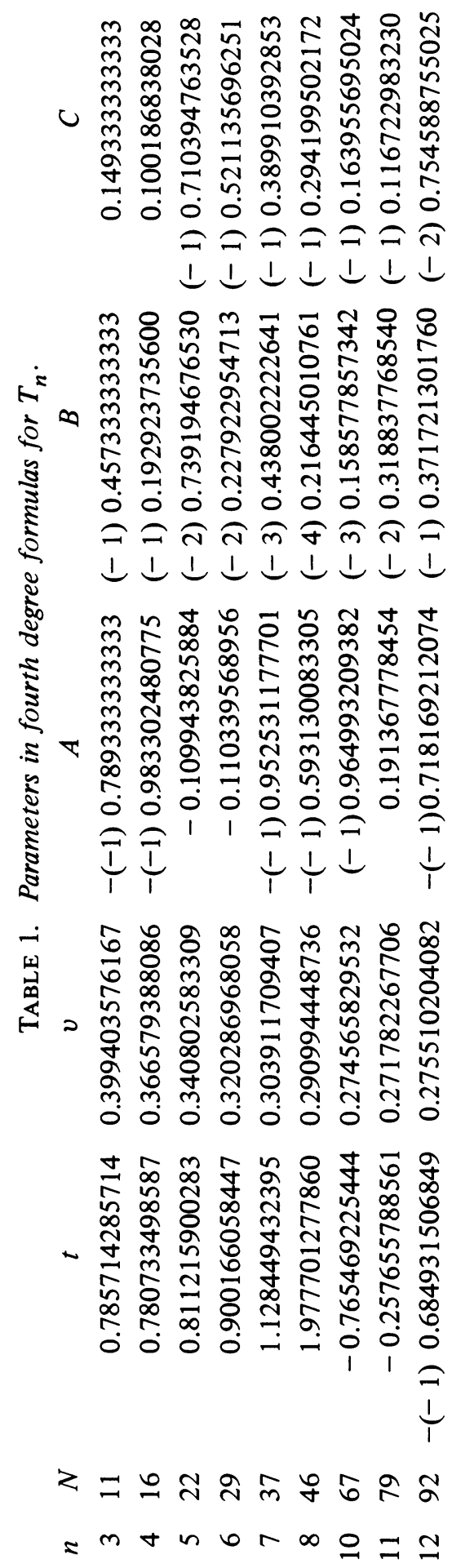


We solve equations (7-1), (7-2), (7-3) for $A, B, C$ in terms of $r, s, t, u, v$. Substituting these expressions into (7-4) and (7-5) and using the fact that

$$
n s+t=1, \quad(n-1) u+2 v=1,
$$

gives two simultaneous equations

$$
g_{1}(n, t, v)=0, \quad g_{2}(n, t, v)=0,
$$

for $t$ and $v$. For a given $n$, if values of $t$ and $v$ can be found to satisfy (8), and if these values are such that the determinant of the linear system for $A, B, C$ (obtained from (7-1), (7-2), (7-3)) is nonzero, then $t, v, A, B, C$ give the desired formula. Table 1 contains numerical values of the parameters for the formulas we found in this way. No formulas were found for $n=9$ and $n \geqslant 13$.

A graph of $1 / t$ as a function of $n$, for the $t$ values of Table 1 , shows that for $n=9,1 / t \simeq 0$. This is an indication that for $n=9$ no formula exists. For $n=3,4$, 5, 6 all the points in the formula lie inside $T_{n}$, but the coefficient $A$ for the centroid is negative. For $n=7,8,10,11,12$ the $n+1$ points with coefficient $B$ lie outside $T_{n}$. For $n=10,11$ all the coefficients are positive.

3. Concluding Remarks. Bykova [1] has given an eleven point fourth degree formula for three dimensional regions which are symmetric with respect to the coordinate planes. $T_{3}$ does not have this symmetry; as far as we know, all the formulas given above are new.

The above calculations were carried out in Fortran double precision (about sixteen significant digits) on the IBM $360 / 65$ at the Data Processing Center at Texas A \& M University. We wish to thank the Department of Mathematics at Texas A \& M University for the computing time for this work.

Department of Mathematics

Texas A \& M University

College Station, Texas 77843

1. T. M. BYKOVA, "Cubature formulas for computing triple integrals which are exact for fourth degree polynomials and have eleven nodes," Vesci A kad. Navuk BSSR Ser Fiz.-Mat. Navuk, no. 1, v. 1970, pp. 51-54. (Russian)

2. R. LAUFFER, "Interpolation mehrfacher Integrale," Arch. Math., v. 6, 1955, pp. 159-164. MR 16, 862 .

3. J. N. LYNESS \& D. JESPERSEN, "Moderate degree symmetric quadrature rules for the triangle," J. Inst. Math. Appl., v. 15, 1975, pp. 19-32.

4. A. H. STROUD, Approximate Calculation of Multiple Integrals, Prentice-Hall Ser. in Automatic Computation, Prentice-Hall, Englewood Cliffs, N. J., 1971. MR 48 \#5348. 